\title{
Antibiotics in the pipeline: a literature review (2017-2020)
}

\author{
Jaffar A. Al-Tawfiq ${ }^{1,2,3,18}$ - Hisham Momattin ${ }^{4} \cdot$ Anfal Y. Al-Ali ${ }^{5} \cdot$ Khalid Eljaaly ${ }^{6,7} \cdot$ Raghavendra Tirupathi $^{8,9,10}$. \\ Mohamed Bilal Haradwala ${ }^{11}$. Swetha Areti ${ }^{12}$. Saad Alhumaid ${ }^{13}$. Ali A. Rabaan ${ }^{14,19}$. Abbas Al Mutair ${ }^{15,16,20}$. \\ Patricia Schlagenhauf ${ }^{17}$
}

Received: 6 August 2021 / Accepted: 23 September 2021 / Published online: 4 October 2021

(c) Springer-Verlag GmbH Germany, part of Springer Nature 2021, corrected publication 2022

\begin{abstract}
Introduction Antimicrobial resistance (AMR) is an emerging global threat. It increases mortality and morbidity and strains healthcare systems. Health care professionals can counter the rising AMR by promoting antibiotic stewardship and facilitating new drug development. Even with the economic and scientific challenges, it is reassuring that new agents continue to be developed.

Methods This review addresses new antibiotics in the pipeline. We conducted a review of the literature including Medline, Clinicaltrials.org, and relevant pharmaceutical companies for approved and in pipeline antibiotics in phase 3 or new drug application (NDA).

Results We found a number of new antibiotics and reviewed their current development status, mode of action, spectra of activity, and indications for which they have been approved. The included studies from phase 3 clinical trials were mainly utilized for the treatment of acute bacterial skin and skin structure infections, community-acquired bacterial pneumonia, and pneumonia acquired in the healthcare settings. The number of these agents is limited against high priority organisms. The identified antibiotics were based mainly on previously known molecules or pre-existing antimicrobial agents.

Conclusion There are a limited number of antibiotics against high priority organisms such as multi-drug-resistant Pseudomonas aeruginosa, and carbapenem-resistant Enterobacteriaceae. New antimicrobial agents directed against the top priority organisms as classified by the World Health Organization are urgently needed.
\end{abstract}

Keywords Antibiotics $\cdot$ Pipeline $\cdot$ Novel antibiotics $\cdot$ New antibiotics

\section{Introduction}

Antibiotics have provided protection against life-threatening bacterial infections for more than a century. However, indiscriminate use of antibiotics and organism evolution have led to the emergence of multi-drug-resistant organisms (MDRO), and at times resistant to most or even all currently available antibiotic classes, extensively drug-resistant or pan-resistant organisms (XDRO, PDRO). Antibiotic resistance is a serious emerging global health threat [1] and certain geographic areas might be affected more than others due to the pattern of antibiotic usage [2]. Thus, there is a great demand to search for novel antibiotics that are effective and safe. Antibiotic development has had several scientific

Jaffar A. Al-Tawfiq

jaffar.tawfiq@jhah.com; jaltawfi@yahoo.com

Extended author information available on the last page of the article and economic challenges over the years. A major hindrance for industrial support for new antimicrobial development is the low return of investment [3]. That said, antibiotics are indispensable for global health. This paper reviews the antibacterial agents launched worldwide since 2017 and details their development status, mode of action, spectra of activity and the indications for which these antibiotics have been approved.

\section{Methodology}

\section{Search strategy}

Two investigators initially reviewed the listed databases and then additional two investigators did a follow-up search to identify new antibiotics in development by searching the FDA, WHO, European medicine agency, and Central Drugs 
Standard Control Organization (India) platforms. Using the 25 new antibiotics identified between January 1st, 2017 and January 31, 2021, we formulated keywords and a search strategy for further databases. Two investigators independently searched electronic databases MEDLINE, NIH U. S. National Library of Medicine (clinicaltrial.gov), and Science Direct for articles as per the Preferred Reporting Items for Systematic Reviews and Meta-Analysis (PRISMA) guidelines for the period January 2017 to November 30th, 2020. When necessary, websites of pharmaceutical companies responsible for the development of the drug were accessed for further relevant information. Only English language articles were selected.

We used the following search terms (Fig. 1): Delafloxacin, Ridinilazole, Afabicin, Gepotidacin,
Meropenem-Vaborbactam, Imipenem-Relebactam, Cefepime "AAI101", Sulbactam-Diazabicyclooctane, Plazomicin, Cefiderocol, Cefilavacin, Nafithromycin, Eravacycline, Lefamulin, Sulopenem Zoliflodacin, Omadacycline, Iclaprim, Solithromycin, Levonadifloxacin, Contezolid, Pretomanid, Taniborbactam, DprE1 inhibitor, Lascufloxacin and the umbrella term "novel antibiotics".

\section{Selection criteria}

Four investigators independently extracted the data from the full text of the selected literature. We selected antibiotics currently in phase III new drug application (NDA), or were FDA approved. We also included drug trials and NDA in China, India, and Japan. We excluded phase I and phase II
Fig. 1 A flow diagram of the search strategy according to the preferred reporting items for systematic review and metaanalysis (PRISMA) guidelines
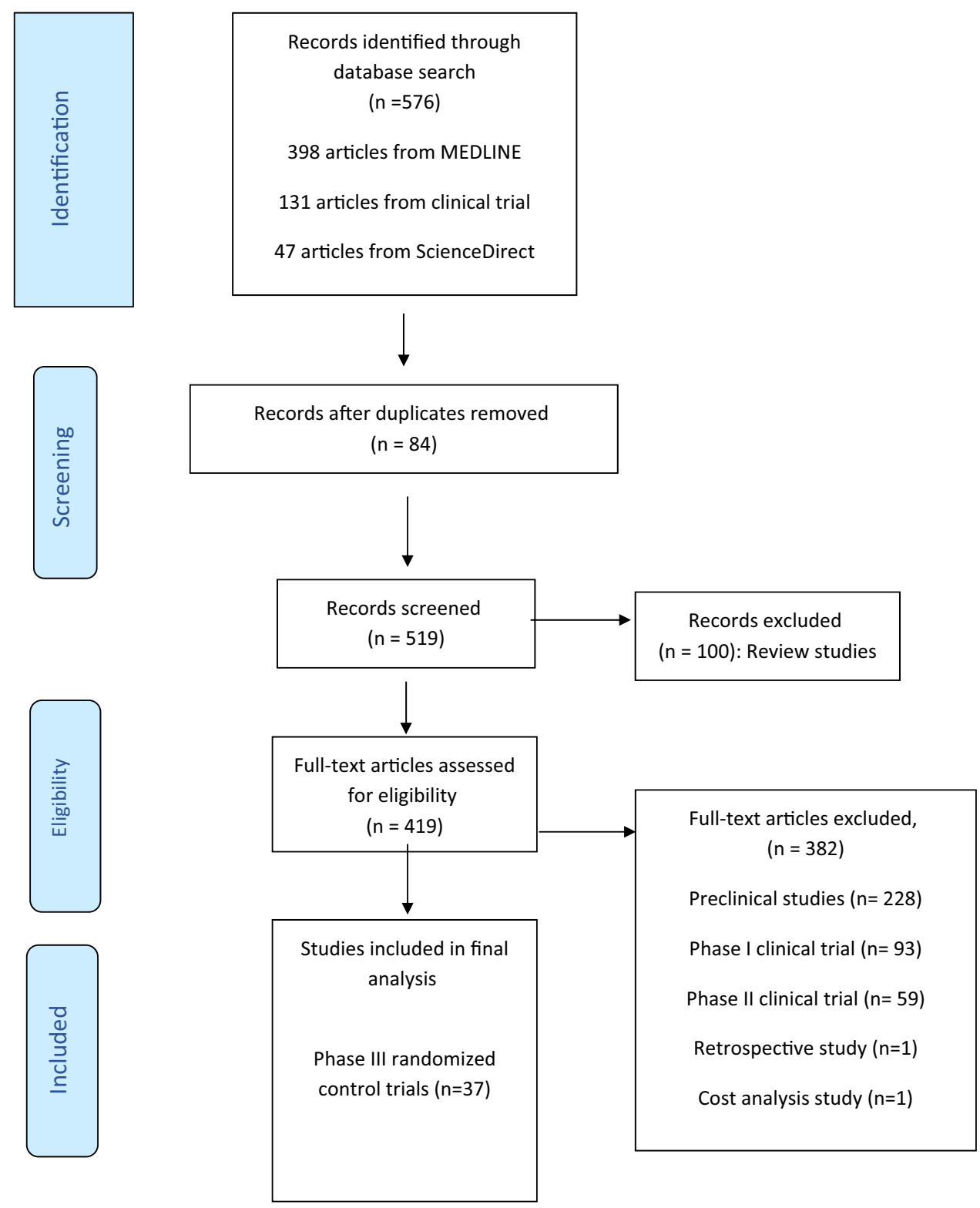

$$
(n=84)
$$

Full-text articles excluded

Preclinical studies $(n=228)$

Phase I clinical trial $(n=93)$

Phase II clinical trial $(n=59)$

ective study $(n=1)$

Cost analysis study $(n=1)$ 
clinical trials, observational studies, case reports, cost analysis studies, and animal models. In the data extraction form, we included method-of-action, spectrum of activity, data from clinical trials, and the included antibiotics' adverse event profiles. Two reviewers assessed the quality of the included literature independently. The information extracted was compared to information provided on the drug manufacturer's website to confirm its development phase. We relied on data published in databases to confirm the spectrum of activity and adverse events.

\section{Results}

Of the $\mathbf{5 7 6}$ articles identified from database searching, 37 phase III clinical trials were included. Table 1 shows a summary of the reviewed antibiotics in the pipeline and their spectrum of activities.

\section{Delafloxacin}

Delafloxacin is a novel anionic fluoroquinolone antibiotic approved by the FDA on June 19th, 2017 [4]. It has the European Medicines Agency's (EMA) approval for the treatment of acute bacterial skin and skin structure infections (ABSSSI) [5]. Delafloxacin inhibits DNA replication, transcription, repair, and recombination by inhibiting the two primary enzymes: DNA gyrase (topoisomerase II) and topoisomerase IV enzymes [6]. Delafloxacin, as other fluoroquinolones, has a high pulmonary concentration of 13:1 compared to plasma and is primarily $(65 \%)$ excreted as unchanged in the urine. Delafloxacin does not seem to prolong the QTc interval on ECG or cause phototoxicity [6]. Similar to other fluoroquinolones, delafloxacin has concentration-dependent antimicrobial activity. It has a low minimum inhibitory concentration (MIC) against Streptococcus pneumoniae and Staphylococcus aureus (including MRSA). Some levofloxacin-resistant S. pneumoniae strains are susceptible to delafloxacin. Delafloxacin is also active against Pseudomonas aeruginosa, anaerobes, and atypical organisms [6].

Delafloxacin was shown to be non-inferior to vancomycin plus aztreonam in the treatment of ABSSSI [7, 8]. In the PROCEED study, 660 adult patients received either delafloxacin $300 \mathrm{mg}$ IV every $12 \mathrm{~h}$ or vancomycin IV $15 \mathrm{mg} /$ $\mathrm{kg}$ every $12 \mathrm{~h}$ plus aztreonam IV $2 \mathrm{~g}$ every $12 \mathrm{~h}$ [7]. In an intent-to-treat (ITT) analysis, the response was similar in both arms (78.2\% in delafloxacin versus $80.9 \%$ in vancomycin + aztreonam arm). Adverse event incidence was higher in the vancomycin plus aztreonam arm (4.5\% versus $0.9 \%)$ [7]. In the second trial, delafloxacin was compared with vancomycin plus aztreonam in 850 adults [8]. In ITT analysis, response was similar in both arms $(83.7 \%$ in delafloxacin arm versus $80.6 \%$ in vancomycin + aztreonam arm) [8]. Vancomycin plus aztreonam had a higher adverse events than delafloxacin (1.2\% versus $2.4 \%$ ) [8]. The efficacy and safety of delafloxacin in community-acquired bacterial pneumonia (CABP) was compared with moxifloxacin in a phase III, multi-center, randomized trial (DEFINE-CABP) [9]. The study showed similar early clinical response of about $89 \%$ in each arm [9].

\section{Meropenem/vaborbactam}

Meropenem/vaborbactam is a fixed combination of meropenem and vaborbactam and was approved by FDA on August 29th, 2017 for treatment of complicated urinary tract infection (cUTI) including pyelonephritis [4] and by EMA on September 20th, 2018 for treatment of cUTI including pyelonephritis, complicated intra-abdominal infection (cIAI), and hospital-acquired bacterial pneumonia (HABP), ventilator-associated bacterial pneumonia (VABP) [10]. Vaborbactam does not have any anti-bacterial activity and its main function is to protect meropenem from degradation by B-lactamases especially Klebsiella pneumoniae carbapenemase (KPC). However, verobactam has no activity against OXA-48 and Metallo- $\beta$-lactamases (MBL) carbapenemases or carbapenem-resistant $P$. aeruginosa [10].

The TANGO I is a phase III randomized clinical trial and it compared meropenem-vaborbactam and piperacillin-tazobactam for 10 days in 550 adult patients with cUTI [11]. The overall success rate was higher in the meropenem/ vaborbactam arm (98.4\% versus $94 \%)$ in a non-inferiority trial. Microbial eradication in the modified ITT analysis occurred in $66.7 \%$ of the meropenem-vaborbactam arm compared with $57.7 \%$ in the piperacillin-tazobactam group [11]. Subsequently, in the TANGO II phase 3 randomized clinical trial, the study evaluated the efficacy and safety of meropenem-vaborbactam in 77 adults with carbapenemresistant Enterobacteriaceae (CRE) confirmed or suspected infections versus best available therapy (BAT) [12]. In microbiologic CRE-modified ITT, meropenem-vaborbactam achieved a higher clinical cure with no difference in microbiologic cure at the end of therapy [12]. There was no significant difference in the mortality at day $28(15.6 \%$ versus $33.3 \% P=0.20)$. Meropenem-vaborbactam had fewer adverse events (84\% versus $92 \%$ ) and fewer renal adverse event (4\% versus $24 \%$ ) [12]. The efficacy and safety evaluations of meropenem-vaborbactam in adults with HABP or VABP are expected to be completed in December 2020 in a phase IIIb TANGO III randomized clinical trial [13].

\section{Imipenem-cilastatin + relebactam}

Imipenem-cilastatin + relebactam is a fixed combination of imipenem, cilastatin, an imipenem renal metabolism 


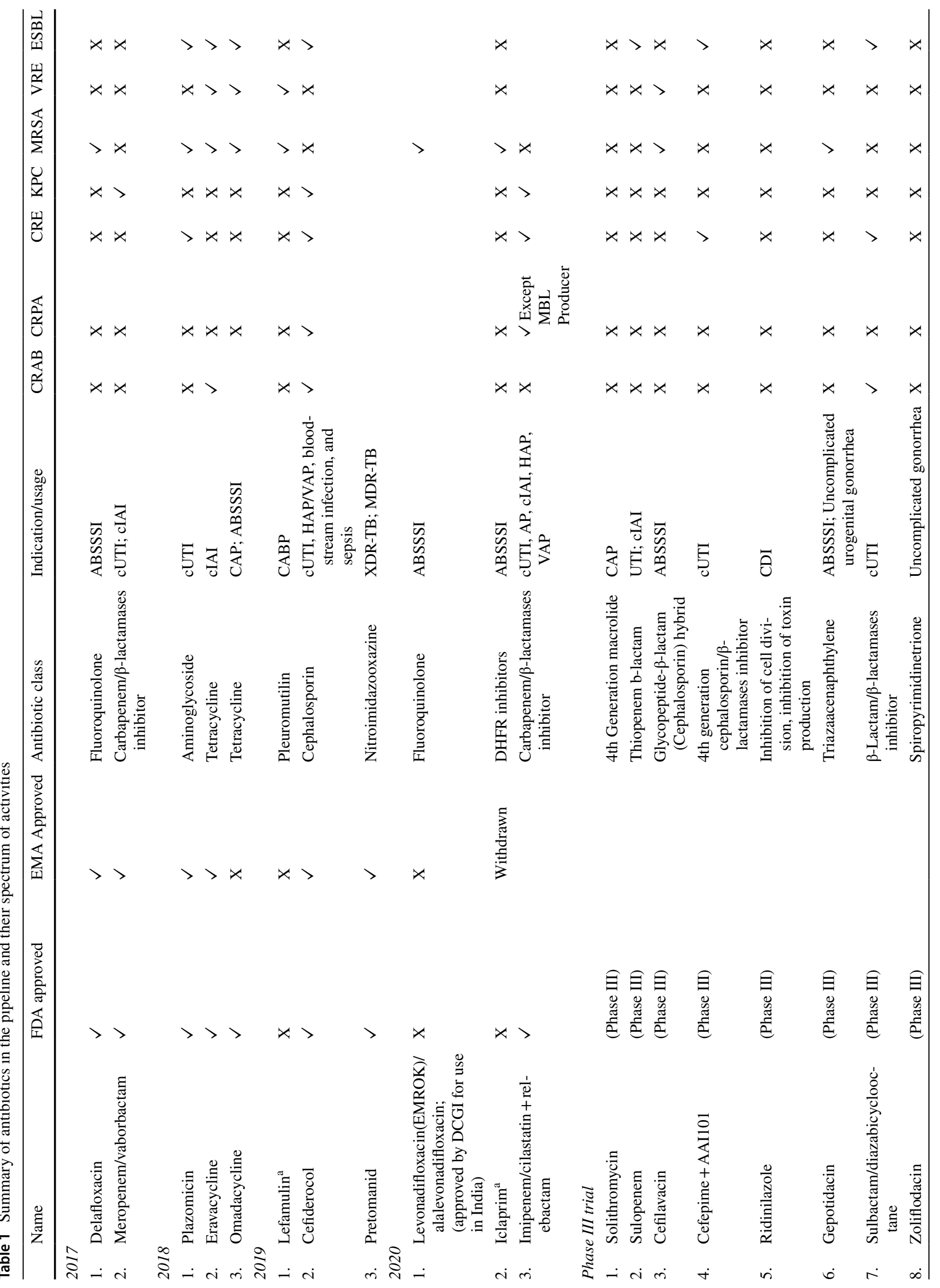


inhibitor, and relebactam, a b-lactamase inhibitor. Relebactam is active against Class A (including ESBL and $\mathrm{KPC}$ ) and Class $\mathrm{C}(\mathrm{AmpC})$ b-lactamases, has activity against ESBL-producing Enterobacteriaceae, KPC, CRE, and possibly carbapenem-resistant $P$. aeruginosa. It has no activity against MBL and OXA-48 producers, Acinetobacter baumannii, or Stenotrophomonas maltophilia [14].

RESTORE-IMI 1 was a phase III randomized trial in 47 adults with HAP/VAP, cIAI, and cUTI caused by Gram-negative imipenem-resistant organisms [15-17]. The treated patients received either imipenem + cilastatin + relebactam or colistin base activity (CBA) plus imipenem + cilastatin. In supplemental microbiological modified ITT, the overall response was $75 \%$ in imipenem/ relebactam group compared with $76.9 \%$ in colistin/imipenem group (difference $-4.5 ; 95 \%$ CI -24.2 to 20.7). All-cause 28-day mortality in imipenem/relebactam compared with colistin/imipenem was $10.7 \%$ versus $23.1 \%$, respectively [15].

RESTORE-IMI 2 trial was completed on April 3rd, 2019 and was a phase III clinical trial comparing imipenem + cilastatin + relebactam with piperacillin + tazobactam in 536 adults with HAP/VAP and all patients also received linezolid. Imipenem/cilastatin/relebactam was non-inferior $(P<0.001)$ to piperacillin/tazobactam when comparing 28 -day all-cause mortality ( $15.9 \%$ vs. $21.3 \%)$ and showed a favorable clinical response $(61.0 \%$ and $55.8 \%)$ at early follow-up [18].

\section{Plazomicin}

Plazomicin is a novel aminoglycoside antibiotic approved by the FDA on June 25th, 2018 and by the EMA on March 19th, 2018 to treat cUTI. Plazomicin binds to the 30 S ribosomal subunit and inhibits protein synthesis in a concentration-dependent manner. It has activity against ESBL-producing Enterobacteriaceae, CRE, MRSA, and organisms producing aminoglycoside-modified enzymes. Like other aminoglycosides, plazomicin is associated with nephrotoxicity, ototoxicity and fetal harm in pregnant women [19].

A phase III randomized trial compared plazomicin with meropenem in 609 adults with cUTI or acute pyelonephritis followed by optional oral therapy. The microbiologic eradication rate at test-of-cure (TOC) visit was higher in plazomicin group vs. meropenem group $(81.7 \%$ vs $70.1 \%$; $95 \%$ CI 2.7-20.3) [20]. In Phase III open-label, non-inferiority (CARE) trial, 39 adults with bloodstream infection, HABP, or VABP due to CRE were treated with plazomicin and allcause mortality at day 28 or significant complications was $23.5 \%$ in the plazomicin arm compared with $50 \%$ of the patients in colistin arm [21]. 


\section{Eravacycline}

Eravacycline is a novel fluorocycline of the tetracyclines group and as such inhibits bacterial protein synthesis by binding to $30 \mathrm{~S}$ ribosomal subunit. Eravacycline overcomes tetracycline-efflux and ribosomal protection mechanism. Eravacycline has activity against MRSA, VRE, ESBL Enterobacteriaceae, A. baumannii, and CRE, but has no activity against $P$. aeruginosa and Burkholderia cenocepacia [22, 23]. In a phase III, randomized, double-blind (IGNITE 1) clinical trial, an MITT showed a clinical cure of $87 \%$ in the eravacycline arm compared to $88.8 \%$ in the ertapenem arm [23]. Microbiological cure was $91.4 \%$ in the eravacycline group versus $95 \%$ in ertapenem group [23]. In the IGNITE4, a second phase III clinical trial, he clinical cure was $90.8 \%$ compared to $91.2 \%$ in the eravacycline and meropenem groups, respectively [24].

\section{Omadacycline}

Omadacycline is a tetracycline antibiotic and was approved in 2018 for treatment of CABP and ABSSSI. It overcomes the resistance by tetracycline-efflux and ribosomal protection mechanisms and has activity against Legionella pneumophila, Mycoplasma pneumoniae, and Chlamydia pneumoniae [25-27]. Thus, it can be used as a single agent to treat $\mathrm{CABP}$ as an alternative to the empirical combination of beta-lactam and macrolide.

The efficacy and safety of omadacycline were tested on a phase III randomized control trial (OPTIC) comparing omadacycline in 388 patients with moxifloxacin in 386 patients with CABP followed by oral omadacycline or Moxifloxacin [28]. The early clinical response was $81.1 \%$ in omadacycline group compared with $82.7 \%$ in the comparator group [28]. In the post-treatment evaluation, clinical response rate was $87.6 \%$ in omadacycline compared with $85.1 \%$ in moxifloxacin arm [28]. The rate of adverse events leading to treatment discontinuation was $5.5 \%$ in omadacycline compared with $7 \%$ in moxifloxacin [28].

Omadacycline had been tested in 316 patients and linezolid in 311 patients with ABSSSI in phase 3 randomized controlled (OASIS-1) trial of either omadacycline or linezolid followed by oral omadacycline or linezolid [29]. In the MITT, the early response rate was $84.8 \%$ vs. $85.5 \%$, respectively, compared with linezolid. [29]. The clinical response of omadacycline was $83 \%$ for MRSA compared with $86 \%$ in the linezolid arm. Treatment-related adverse events were $18 \%$ in omadacycline arm versus $18.3 \%$ with linezolid [29].

\section{Lefamulin}

Lefamulin is a novel pleuromutilin antibiotic and was approved in August 2019 by U.S. FDA for use in CABP.
Lefamulin inhibits protein synthesis by inhibition of $50 \mathrm{~S}$ bacterial ribosome. It has activity against $S$. pneumoniae, MRSA, VRE, MDR Neisseria gonorrhoeae, Chlamydophila pneumonia, L. pneumophila, M. pneumoniae, and Haemophilus influenzae. Lefamulin has a time-dependent killing with higher concentrations in epithelial lining fluid than in plasma [30]. Lefamulin was non-inferior to moxifloxacin in 551 adults with CABP in a phase III (LEAP-1) clinical trial [31]. Early clinical response was $87.3 \%$ versus $90.2 \%$ [31]. The rate of drug discontinuation was $2.9 \%$ in the lefamulin arm and $4.4 \%$ in the moxifloxacin arm [31]. In the second phase III clinical trial (LEAP-2), oral lefamulin was compared to moxifloxacin in 738 patients with CABP [32]. Lefamulin was non-inferior to moxifloxacin for CABP $(90.8 \%$ versus $90.8 \%$ ), clinical response $(87.5 \%$ versus $89.1 \%)$, and clinically evaluable population (89.7\% versus $93.6 \%$ ) [32].

\section{Cefiderocol}

Cefiderocol is the first in a class of siderophore cephalosporins with activity against carbapenemase-producing Gram-negative bacteria (CRE, CRPA, and CRAB), MDR S. maltophilia, and Burkholderia cepacia, and ESBL- and MBL-producing organisms. Potential indications include cUTI, HAP/VAP, bloodstream infection, and sepsis caused by MDR Gram-negative isolate [33, 34]. A phase III trial compared cefiderocol in APEKS-NP trial with meropenem in 300 adults with healthcare-associated pneumonia (HCAP), HABP, or VABP with all-cause mortality at day 14 of $12.4 \%$ vs. $11.6 \%$, respectively [35]. The second Phase III clinical trial (CREDIBLE-CR) will compare cefiderocol with best available therapy in 150 adults with HAP, VAP, HCAP, cUTI, or BSI/Sepsis caused by carbapenem-resistant Gram-negative pathogens [36].

\section{Levonadifloxacin/alalevonadifloxacin}

Levonadifloxacin and its prodrug, alalevonadifloxacin, are broad-spectrum benzoquinolizine sub-class of quinolones and are active against multi-drug-resistant Gram-positive pathogens including MRSA, hVISA, VRSA, and quinoloneresistant strains $[36,37]$. A phase III clinical trial compared oral/IV levonadifloxacin to oral/IV Linezolid in a multicenter, randomized, open-label trial [38]. Clinical cure rates for levonadifloxacin were higher compared to linezolid in the IV sub-group (91.0\% versus $87.8 \%$ ) and in the oral subgroup (95.2\% versus $93.6 \%$ ) [38].

\section{Pretomanid}

Pretomanid is a nitroimidazooxazine antibiotic [37] and is being proposed for use in a combination regimen to treat adults with pulmonary XDR-TB, or treatment-intolerant or nonresponsive MDR-TB [39]. It exhibits both 
mycobactericidal activity against replicating and static $M$. tuberculosis [39]. In a phase III trial, 11 patients (10\%) from 109 had an unfavorable outcome (7 deaths, 2 relapses, 1 lost to follow-up, and 1 withdrawal of treatment) [40].

\section{Iclaprim}

Iclaprim is a dihydrofolate reductase inhibitor (DHFR) which inhibits bacterial nucleic acid and protein synthesis and has superior activity to trimethoprim and overcomes trimethoprim resistance. It is bactericidal against Gram-positive MDR bacteria. Two phase III trials (ASSSIST 1 and 2) compared IV iclaprim and IV linezolid in the treatment of complicated ABSSSI. These studies failed to show noninferiority of iclaprim and caused QT-prolongation [41, 42]. A two-phase III clinical trial (REVIVE-1 and 2) assessed iclaprim non-inferiority against vancomycin in ABSSSI $[43,44]$. In REVIVE-1, early clinical response was $83.5 \%$ and $79.7 \%$ to iclaprim and vancomycin, respectively. In REVIVE-2, the clinical response was $82.7 \%$ in the iclaprim arm and $76.3 \%$ in the vancomycin arm [43, 44].

\section{Sulopenem}

Sulopenem is a novel thiopenem B-lactam antibiotic, developed as a prodrug of sulopenem-etzadroxil for therapy of UTI and cIAI. It has good activity against ESBL-producing Enterobacteriaceae [45, 46]. The first Phase III clinical trial (SURE 1) will evaluate the efficacy and safety of PO sulopenem-etzadroxil/probenecid versus ciprofloxacin PO in women with uncomplicated UTI [47]. The second ongoing phase III (SURE 2) clinical trial is comparing sulopenem IV followed by sulopenem-etzadroxil/probenecid PO versus ertapenem IV followed by ciprofloxacin PO or amoxicillinclavulanate PO in adults with cUTI [48]. The SURE 3 trial is the third, ongoing, phase III clinical trial comparing sulopenem IV followed by sulopenem-etzadroxil/probenecid PO to ertapenem IV followed by ciprofloxacin PO or amoxicillin-clavulanate PO in adults with cIAI [49].

\section{Contezolid}

Contezolid (MRX-I) is an oxazolidinone and is being considered for the treatment of complicated skin and soft tissue infections (cSSTI) caused by resistant Gram-positive bacteria [50]. It has activity against MRSA, vancomycin-resistant $E$. faecium, and resistant S. pneumoniae. A Phase II trial of contezolid acefosamil in patients with ABSSSI has been completed (NCT02269319) in the United States by MicuRx, and compared MRX-I (Contezolid) with vancomycin [50]. In a phase III clinical trial, contezolid was non-inferior $(93.0 \%)$ compared to linezolid (93.4\%) for the clinical cure rate of patients with cSSSIs [51].

\section{Solithromycin}

Solithromycin is a novel, fourth-generation macrolide, known as fluoroketolide, which inhibits protein synthesis by binding to bacterial ribosome. It has activity against macrolideresistant S. pneumonia, H. Influenzae, and atypical pathogens with potential indication for use in CABP [52, 53]. Oral solithromycin was non-inferior to oral moxifloxacin in 860 adults with CABP in a phase III trial (SOLITAIRE-ORAL) [54]. Early clinical response was $78.2 \%$ versus $77.9 \%$ Elevation of ALT was observed in 5.4\% of the solithromycin group compared with $3.3 \%$ in moxifloxacin group and elevated AST in $2.5 \%$ of solithromycin group compared with $1.9 \%$ in the comparator group [54]. Solithromycin (IV to PO) was noninferior to moxifloxacin (IV to PO)in 863 adults with CABP in a phase III clinical trial (SOLITAIRE-IV) [55]. The early clinical response in ITT was $79.3 \%$ in solithromycin arm compared with $79.7 \%$ in moxifloxacin arm with a higher rate of adverse drug reactions in the solithromycin arm [55]. The efficacy of $\mathrm{PO}$ solithromycin compared with standard treatment (IV ceftriaxone with oral azithromycin) in 264 adults with uncomplicated gonorrhea has been tested in a phase III clinical trial (SOLITAIRE-U) [56]. The cure rate was 80.5\% in solithromycin arm compared with $84.5 \%$ in ceftriaxone/ azithromycin arm [56].

\section{Cefepime + AAl101 (Enmetazobactam)}

Cefepime + AAI101 is a B-lactam and B-lactamase inhibitor [57]. AAI101 is a novel inhibitor of ESBL, and classes A and D carbapenemases [57, 58]. The addition of AAI101 to cefepime resulted in a significant in vivo reduction in the MIC50 against Enterobacteriaceae isolates [57]. Cefepime/ AAI101 is being studied in a phase III randomized clinical trial in cUTI adults in comparison with piperacillin/ tazobactam. The study was started on September 24, 2018 and completed on January 30th, 2020 (Clinical registration NCT03687255) [59].

\section{Ridinilazole}

Ridinilazole is a novel, non-absorbable, oral antimicrobial and is restricted to the gastrointestinal tract. An in vitro study showed that ridinilazole is a potent inhibitor of $C$. difficile by inhibiting cell division and reducing toxin production [60, 61]. Ridinilazole is being studied in a phase III randomized, controlled trial in comparison with fidaxomicin in one study (Clinical registration NCT02784002) [62] and with vancomycin (Clinical registration NCT03595566) in another study [63] for the treatment of $C$. difficile infection. 


\section{Gepotidacin}

Gepotidacin (GSK2140944) is a novel triazaacenaphthylene antimicrobial agent and is an inhibitor of bacterial type II topoisomerase [64]. It shows excellent activity against MDR N. gonorrhoeae and Gram-positive bacteria including MRSA [65]. It had undergone phase II trial for the treatment of uncomplicated urogenital gonorrhea (Clinical registration NCT02294682) [66] and in treatment of ABSSSI caused by Gram-positive bacteria (Clinical registration NCT02045797) [67]. It is currently being used in a phase III, randomized study comparing efficacy and safety of Gepotidacin to Nitrofurantoin in uncomplicated urinary tract infection (Clinical registration-NCT04020341) [68].

\section{Sulbactam/Diazabicyclooctane}

Sulbactam/Diazabicyclooctane is a combination of B-lactam/B-lactamase inhibitors with a wide range of B-lactamases inhibition, including class A, C, D, CRE, and CRAB [69]. A Phase III randomized study will evaluate efficacy and safety of intravenous Sulbactam-ETX2514 in treating patients with A. baumannii-calcoaceticus Complex infection (Clinical registration NCT03894046) [70].

\section{Zoliflodacin}

Zoliflodacin is a novel Spiropyrimidinetrione is a type II topoisomerase inhibitor with a good activity against MDR $N$. gonorrhea [71]. Another non-inferiority phase III clinical trial is evaluating the safety and efficacy of zoliflodacin vs. a combination of ceftriaxone and azithromycin for the treatment of uncomplicated gonorrhea (Clinical registration NCT03959527) [72].

\section{Taniborbactam}

Taniborbactam is an injectable, beta-lactamase inhibitor. Taniborbactam (VNRX-5133) can inhibit metallo-beta-lactamase and serine-beta-lactamases and has a broad-spectrum inhibitory activity against Ambler Class A (ESBLs), $\mathrm{B}$ (NDM and VIM), C (AmpC from P. aeruginosa) and, to a lesser extent, D (OXA) $\beta$-lactamase [72]. It is currently undergoing phase III clinical trial (ClinicalTrials.govNCT03840148) comparing cefepime-taniborbactam to meropenem in adults with cUTI [73].

\section{Discussion}

The current review details the clinical outcome of the use of the novel antibiotics in the pipeline. We found that the "novel" antibiotics were often based on previously known molecules or pre-existing antimicrobial agents. With regard to the spectra of activity, there are a limited number of new antibiotics against high priority organisms such as multidrug-resistant $P$. aeruginosa, and carbapenem-resistant Enterobacteriaceae. These antibiotics include: plazomicin [21], eravacycline [22, 23], imipenem-cilastatin + relebactam [14-18], Sulbactam/Diazabicyclooctane [70] and Cefiderocol [33-36]. This is an important deficit as the acute shortage is particularly challenging for the "priority organisms". The issue of the need for new antimicrobial agents for priority organisms had been on the radar of the World Health Organization (WHO) for some time. The WHO priority list includes: 1) three "critical" organisms (A. baumannii, carbapenem-resistant $P$. aeruginosa, carbapenem-resistant and 3rd-generation cephalosporin-resistant Enterobacteriaceae); 2) six "high priority" organisms (Enterococcus faecium, $S$. aureus (vancomycin-resistant, methicillin-resistant, vancomycin intermediate and resistant), clarithromycin-resistant Helicobacter pylori, fluoroquinolone-resistant Campylobacter, fluoroquinolone-resistant Salmonella spp., 3rd-generation cephalosporin-resistant fluoroquinolone-resistant $N$. gonorrhoeae; and 3) three "medium priority" organisms (penicillin-non-susceptible $S$. pneumoniae, ampicillin-resistant $H$. influenzae, fluoroquinolone-resistant Shigella spp.) [74]. There is still a need to have further studies addressing these priority organisms as suggested by the WHO especially multi-drug-resistant tuberculosis and Gram-negative bacteria [75]. Of the included studies, few studies addressed healthcare-associated pneumonia. However, the burden of drug-resistant bacteria is high among these types of infections. This is an added issue to the clinical trial for the therapy of MDRO or pan-drug-resistant organism therapy. One of the issues of such trials is the use of strict definitions and the need to include one source of infection such as bloodstream infection [76]. Another difficulty is the difficulty in recruiting patients with MDRO. One study showed that out of the 2100 screened patients only 37 patients were randomized [77]. It had been suggested that such trials should include observational studies that are planned and executed in a way to reduce bias in the search of therapy for MDRO [76]. How scientists could run such observational studies giving the different predictors of mortality including the site of infection, gender, comorbidities and the implicated organism is an important question to address [78].

The strength of our review is the thorough and comprehensive searches of platforms, clinical trial registries, databases and pharmaceutical company websites. The review is limited by the heterogenicity of included studies and difficulties in the quantification of comparator arms. The regulatory requirements for the registration of antibiotics vary between the US and the EU and some of the drug approval status may quickly change. However, the review highlights a pipeline paucity of these essential drugs. This problem 
may be further enhanced by the current COVID-19 pandemic where there may be lack of economic support for developing new agents reinforcing the need for international cooperation and coordination [79]. There is also a concern regarding the increased costs of these new antimicrobial agents for the treatment of MDR-organisms. In one study, the estimated cost for the treatment of methicillin-resistant $S$. aureus bacteremia is 6-60 times the cost of older antibiotics and that for carbapenem-resistant Enterobacterales or MDR $P$. aeruginosa or carbapenem-resistant A. baumannii is 2-20 times that of the older medications [80]. Clearly, there must be incentives for the industry to develop novel antibiotics and $R \& D$ incentive strategies ranging from single rewards to complex international models are needed to push future development. There is no alternative-when the pipeline runs dry, MDR-organisms will rule the world.

\section{Declarations}

Conflict of interest All the authors have no conflicts of interest.

\section{References}

1. Aslam B, Wang W, Arshad MI, Khurshid M, Muzammil S, Rasool $\mathrm{MH}$, et al. Antibiotic resistance: a rundown of a global crisis. Infect Drug Resist. 2018;11:1645. https://doi.org/10.2147/IDR. S173867.

2. Al-Tawfiq JA, Stephens G, Memish ZA. Inappropriate antimicrobial use and potential solutions: a Middle Eastern perspective. Expert Rev Anti Infect Ther. 2010;8:765-74. https://doi.org/10. 1586/eri.10.56.

3. Towse A, Hoyle CK, Goodall J, Hirsch M, Mestre-Ferrandiz $\mathrm{J}$, Rex JH. Time for a change in how new antibiotics are reimbursed: development of an insurance framework for funding new antibiotics based on a policy of risk mitigation. Health Policy 2017;121:1025-30. https://doi.org/10.1016/J.HEALTHPOL.2017. 07.011 .

4. FDA. Novel drug approvals for 2017 n.d. https://www.fda.gov/ drugs/new-drugs-fda-cders-new-molecular-entities-and-new-thera peutic-biological-products/novel-drug-approvals-2017. Accessed 20 Nov 2020.

5. Medicines Agency E. European Medicines Agency decision $\mathrm{P} / 0148 / 2018$ of 18 May 2018 on the review of a granted waiver for delafloxacin (EMEA-001080-PIP01-10). n.d.

6. Jorgensen SCJ, Mercuro NJ, Davis SL, Rybak MJ. Delafloxacin: place in therapy and review of microbiologic, clinical and pharmacologic properties. Infect Dis Ther. 2018;7:197-217. https:// doi.org/10.1007/s40121-018-0198-x.

7. Pullman J, Gardovskis J, Farley B, Sun E, Quintas M, Lawrence L, et al. Efficacy and safety of delafloxacin compared with vancomycin plus aztreonam for acute bacterial skin and skin structure infections: A Phase 3, double-blind, randomized study. J Antimicrob Chemother. 2017;72:3471-80. https://doi.org/10.1093/jac/ dkx329.

8. O'Riordan W, McManus A, Teras J, Poromanski I, Cruz-Saldariagga M, Quintas M, et al. A Comparison of the efficacy and safety of intravenous followed by oral delafloxacin with vancomycin plus aztreonam for the treatment of acute bacterial skin and skin structure infections: a phase 3, multinational, double-blind, randomized study. Clin Infect Dis. 2018;67:657-66. https://doi. org/10.1093/cid/ciy165.

9. Horcajada JP, Salata RA, Álvarez-Sala R, Nitu FM, Lawrence L, Quintas M, et al. A phase 3 study to compare delafloxacin with moxifloxacin for the treatment of adults with communityacquired bacterial pneumonia (Define-CABP). Open Forum Infect Dis. 2020. https://doi.org/10.1093/ofid/ofz514.

10. Dhillon S. Meropenem/vaborbactam: a review in complicated urinary tract infections. Drugs. 2018;78:1259-70. https://doi.org/10. 1007/s40265-018-0966-7.

11. Kaye KS, Bhowmick T, Metallidis S, Bleasdale SC, Sagan OS, Stus V, et al. Effect of meropenem-vaborbactam vs piperacillintazobactam on clinical cure or improvement and microbial eradication in complicated urinary tract infection the TANGO I randomized clinical trial. JAMA. 2018;319:788-99. https://doi.org/ 10.1001/jama.2018.0438.

12. Wunderink RG, Giamarellos-Bourboulis EJ, Rahav G, Mathers AJ, Bassetti M, Vazquez J, et al. Effect and safety of meropenemvaborbactam versus best-available therapy in patients with carbapenem-resistant enterobacteriaceae infections: the TANGO II randomized clinical trial. Infect Dis Ther. 2018;7:439-55. https:// doi.org/10.1007/s40121-018-0214-1.

13. Melinta Therapeutics I. A study of Meropenem-Vaborbactam versus Piperacillin/Tazobactam in participants with hospitalacquired and ventilator-associated bacterial pneumonia-full text view-ClinicalTrials.gov 2016. https://clinicaltrials.gov/ct2/show/ NCT03006679. Accessed 28 Nov 2020.

14. Zhanel GG, Lawrence CK, Adam H, Schweizer F, Zelenitsky $\mathrm{S}$, Zhanel $\mathrm{M}$, et al. Imipenem-relebactam and meropenemvaborbactam: two novel carbapenem- $\beta$-lactamase inhibitor combinations. Drugs. 2018;78:65-98. https://doi.org/10.1007/ s40265-017-0851-9.

15. Kaye K, File T, Boucher HW, Brown M, Aggrey A, Khan I, et al. 1339. Results for the supplemental microbiological modified intent-to-treat (SmMITT) population of the RESTORE-IMI 1 trial of imipenem/cilastatin/relebactam (IMI/REL) vs. imipenem/ cilastatin plus colistin (IMI+CST) in patients with Imipenemnonsusceptible (NS) bacterial infections. Open Forum Infect Dis. 2018;5:S409-S409. https://doi.org/10.1093/ofid/ofy210.1171.

16. Brown ML, Motsch J, Kaye KS, File TM, Boucher HW, Vendetti $\mathrm{N}$, et al. Evaluation of renal safety between imipenem/relebactam and colistin plus imipenem in patients with imipenem-nonsusceptible bacterial infections in the randomized, Phase 3 RESTOREIMI 1 Study. Open Forum Infect Dis. 2020. https://doi.org/10. 1093/ofid/ofaa054.

17. Kaye KS, Boucher HW, Brown ML, Aggrey A, Khan I, Joeng HK, et al. Comparison of treatment outcomes between analysis populations in the RESTORE-IMI 1 phase 3 trial of imipenem-cilastatinrelebactam versus colistin plus imipenem-cilastatin in patients with imipenem-nonsusceptible bacterial infections. Antimicrob Agents Chemother. 2020. https://doi.org/10.1128/AAC.02203-19.

18. Titov I, Wunderink RG, Roquilly A, Rodríguez Gonzalez D, David-Wang A, Boucher HW, et al. A randomized, double-blind, multicenter trial comparing efficacy and safety of imipenem/ cilastatin/relebactam versus piperacillin/tazobactam in adults with hospital-acquired or ventilator-associated bacterial pneumonia (RESTORE-IMI 2 Study). Clin Infect Dis. 2020. https://doi.org/ $10.1093 / \mathrm{cid} / \mathrm{ciaa} 803$.

19. Shaeer KM, Zmarlicka MT, Chahine EB, Piccicacco N, Cho JC Plazomicin: a next-generation aminoglycoside. Pharmacother J Hum Pharmacol Drug Ther. 2019;39:77-93. https://doi.org/10. 1002/phar.2203.

20. Cloutier DJ, Komirenko AS, Cebrik DS, Keepers TR, Krause KM, Connolly LE, et al. Plazomicin vs. meropenem for complicated urinary tract infection (cUTI) and acute pyelonephritis (AP): 
diagnosis-specific results from the phase 3 EPIC study. Open Forum Infect Dis. 2017;4:S532-S532. https://doi.org/10.1093/ ofid/ofx163.1385.

21. McKinnell JA, Connolly LE, Pushkin R, Jubb AM, O'Keeffe $\mathrm{B}$, Serio AW, et al. Improved outcomes with plazomicin (PLZ) compared with colistin (CST) in patients with bloodstream infections (BSI) caused by carbapenem-resistant enterobacteriaceae (CRE): results from the CARE study. Open Forum Infect Dis. 2017;4:S531-S531. https://doi.org/10.1093/ofid/ofx163.1383.

22. Sutcliffe JA, O'Brien W, Fyfe C, Grossman TH. Antibacterial activity of eravacycline (TP-434), a novel fluorocycline, against hospital and community pathogens. Antimicrob Agents Chemother. 2013;57:5548-58. https://doi.org/10.1128/AAC.01288-13.

23. Solomkin JS, Gardovskis J, Lawrence K, Montravers P, Sway A, Evans D, et al. IGNITE4: results of a phase 3, randomized, multicenter, prospective trial of eravacycline vs meropenem in the treatment of complicated intraabdominal infections. Clin Infect Dis. 2019;69:921-9. https://doi.org/10.1093/cid/ciy1029.

24. Solomkin J, Evans D, Slepavicius A, Lee P, Marsh A, Tsai L, et al. Assessing the efficacy and safety of eravacycline vs ertapenem in complicated intra-abdominal infections in the investigating gramnegative infections treated with eravacycline (IGNITE 1) trial a randomized clinical trial. JAMA Surg. 2017;152:224-32. https:// doi.org/10.1001/jamasurg.2016.4237.

25. Cho JC, Childs-Kean LM, Zmarlicka MT, Crotty MP. Return of the tetracyclines: omadacycline, a novel aminomethylcycline antimicrobial. Drugs Today. 2018;54:209-17. https://doi.org/10.1358/ dot.2018.54.3.2800620.

26. Tanaka SK, Steenbergen J, Villano S. Discovery, pharmacology, and clinical profile of omadacycline, a novel aminomethylcycline antibiotic. Bioorganic Med Chem. 2016;24:6409-19. https://doi. org/10.1016/j.bmc.2016.07.029.

27. Chambers HF. Omadacycline - the newest tetracycline. N Engl J Med. 2019;380:588-9. https://doi.org/10.1056/nejme1900188.

28. Stets R, Popescu M, Gonong JR, Mitha I, Nseir W, Madej A, et al. Omadacycline for community-acquired bacterial pneumonia. N Engl J Med. 2019;380:517-27. https://doi.org/10.1056/nejmo a1800201.

29. O’Riordan W, Green S, Overcash JS, Puljiz I, Metallidis S, Gardovskis J, et al. Omadacycline for acute bacterial skin and skinstructure infections. N Engl J Med. 2019;380:528-38. https://doi. org/10.1056/nejmoa1800170.

30. Veve MP, Wagner JL. Lefamulin: review of a promising novel pleuromutilin antibiotic. Pharmacotherapy. 2018;38:935-46. https://doi.org/10.1002/phar.2166

31. File TM, Goldberg L, Das A, Sweeney C, Saviski J, Gelone SP, et al. Efficacy and safety of intravenous-to-oral lefamulin, a pleuromutilin antibiotic, for the treatment of community-acquired bacterial pneumonia: the phase III lefamulin evaluation against pneumonia (LEAP 1) trial. Clin Infect Dis. 2019;69:1856-67. https://doi.org/10.1093/cid/ciz090.

32. Alexander E, Goldberg L, Das A, Moran GJ, Sandrock C, Gasink LB, et al. LB6. Oral lefamulin is safe and effective in the treatment of adults with community-acquired bacterial pneumonia (CABP): results of lefamulin evaluation against pneumonia (LEAP 2) study. Open Forum Infect Dis. 2018;5:S761-S761. https://doi.org/10. 1093/ofid/ofy229.2180.

33. Kish $\mathrm{T}$. New antibiotics in development target highly resistant gram-negative organisms. Pharm Ther. 2018;43:116-20.

34. Saisho Y, Katsube T, White S, Fukase H, Shimada J. Pharmacokinetics, safety, and tolerability of cefiderocol, a novel siderophore cephalosporin for gram-negative bacteria, in healthy subjects. Antimicrob Agents Chemother. 2018. https://doi.org/10.1128/ AAC.02163-17.

35. Wunderink RG, Matsunaga Y, Ariyasu M, Clevenbergh P, Echols $\mathrm{R}$, Kaye KS, et al. Cefiderocol versus high-dose, extended-infusion meropenem for the treatment of Gram-negative nosocomial pneumonia (APEKS-NP): a randomised, double-blind, phase 3, noninferiority trial. Lancet Infect Dis. 2021;21:213-25. https://doi. org/10.1016/S1473-3099(20)30731-3.

36. Shionogi. Study of S-649266 or best available therapy for the treatment of severe infections caused by carbapenem-resistant gram-negative pathogens-full text view-ClinicalTrials.gov 2016. https://clinicaltrials.gov/ct2/show/NCT02714595. Accessed 13 Dec 2020.

37. Jacobs MR, Bajaksouzian S, Windau A, Appelbaum PC, Patel MV, Gupte SV, et al. In vitro activity of the new quinolone WCK 771 against staphylococci. Antimicrob Agents Chemother. 2004;48:3338-42. https://doi.org/10.1128/AAC.48.9.3338-3342. 2004.

38. Bhatia A, Mastim M, Shah M, Gutte R, Joshi P, Kumbhar D, et al. Efficacy and safety of a novel broad-spectrum anti-MRSA agent levonadifloxacin compared with linezolid for acute bacterial skin and skin structure infections: a phase 3, openlabel, randomized study. J Assoc Physicians India. 2020;68:30-6.

39. Stover CK, Warrener P, VanDevanter DR, Sherman DR, Arain $\mathrm{TM}$, Langhorne $\mathrm{MH}$, et al. A small-molecule nitroimidazopyran drug candidate for the treatment of tuberculosis. Nature. 2000;405:962-6. https://doi.org/10.1038/35016103.

40. Conradie F, Diacon AH, Ngubane N, Howell P, Everitt D, Crook AM, et al. Treatment of highly drug-resistant pulmonary tuberculosis. N Engl J Med. 2020;382:893-902. https://doi.org/10.1056/ nejmoa1901814.

41. Huang DB, Strader CD, MacDonald JS, VanArendonk M, Peck R, Holland T. An updated review of iclaprim: a potent and rapidly bactericidal antibiotic for the treatment of skin and skin structure infections and nosocomial pneumonia caused by gram-positive including multidrug-resistant bacteria. Open Forum Infect Dis. 2018;5:ofy003. https://doi.org/10.1093/ofid/ofy003.

42. Abbas M, Paul M, Huttner A. New and improved? A review of novel antibiotics for Gram-positive bacteria. Clin Microbiol Infect. 2017;23:697-703. https://doi.org/10.1016/j.cmi.2017.06. 010 .

43. Huang DB, Corey GR, Holland TL, Lodise T, O'Riordan W, Wilcox MH, et al. Pooled analysis of the phase 3 REVIVE trials: randomised, double-blind studies to evaluate the safety and efficacy of iclaprim versus vancomycin for treatment of acute bacterial skin and skin-structure infections. Int $\mathbf{J}$ Antimicrob Agents. 2018;52:233-40. https://doi.org/10.1016/j.ijantimicag.2018.05. 012.

44. Noviello S, Ralph Corey G, Holland TL, Lodise T, O'Riordan $\mathrm{W}$, Wilcox $\mathrm{MH}$, et al. A pooled analysis of patients with wound infections in the Phase 3 REVIVE trials: randomized, doubleblind studies to evaluate the safety and efficacy of iclaprim versus vancomycin for treatment of acute bacterial skin and skin structure infections. J Med Microbiol. 2020;69:625-30. https://doi.org/10. 1099/jmm.0.001177.

45. Karlowsky JA, Adam HJ, Baxter MR, Denisuik AJ, Lagacé-Wiens PRS, Walkty AJ, et al. In vitro activity of sulopenem, an Oral Penem, against urinary isolates of Escherichia coli. Antimicrob Agents Chemother. 2019. https://doi.org/10.1128/AAC.01832-18.

46. Puttagunta S, Aronin S, Huband M, Flamm RK, Dunne M. 1363. Sulopenem activity against enterobacteriaceae isolates from patients with urinary tract infection or intra-abdominal infection. Open Forum Infect Dis. 2018;5:S417-S417. https://doi.org/10. 1093/ofid/ofy210.1194.

47. Iterum Therapeutics IL. Oral sulopenem-etzadroxil/probenecid versus ciprofloxacin for uncomplicated urinary tract infection in adult women-full text view-ClinicalTrials.gov 2017. https:// clinicaltrials.gov/ct2/show/NCT03354598. Accessed 14 Dec 2020. 
48. Iterum Therapeutics IL. Sulopenem followed by sulopenemetzadroxil/probenecid vs ertapenem followed by cipro for complicated UTI in adults-full text view-ClinicalTrials.gov. 2017 n.d. https://clinicaltrials.gov/ct2/show/NCT03357614. Accessed 14 Dec 2020.

49. Iterum Therapeutics IL. Sulopenem versus ertapenem for complicated intra-abdominal infection (cIAI) - full text view-ClinicalTrials.gov 2017. https://clinicaltrials.gov/ct2/show/NCT03 358576. Accessed 14 Dec 2020.

50. Wu J, Cao G, Wu H, Chen Y, Guo B, Wu X, et al. Evaluation of the effect of contezolid (MRX-I) on the corrected QT interval in a randomized, double-blind, placebo- And positive-controlled crossover study in healthy Chinese volunteers. Antimicrob Agents Chemother. 2020. https://doi.org/10.1128/AAC.02158-19.

51. MicuRx reports favorable results of phase 3 trial of contezolid in China n.d. https://www.ns-healthcare.com/news/micurx-conte zolid-china/. Accessed 8 Sept 2021.

52. Donald BJ, Surani S, Deol HS, Mbadugha UJ, Udeani G. Spotlight on solithromycin in the treatment of community-acquired bacterial pneumonia: design, development, and potential place in therapy. Drug Des Dev Ther. 2017;11:3559-66. https://doi.org/10.2147/ DDDT.S119545.

53. Buege MJ, Brown JE, Aitken SL. Solithromycin: a novel ketolide antibiotic. Am J Heal Pharm. 2017;74:875-87. https://doi.org/10. 2146/ajhp160934.

54. Barrera CM, Mykietiuk A, Metev H, Nitu MF, Karimjee N, Doreski PA, et al. Efficacy and safety of oral solithromycin versus oral moxifloxacin for treatment of community-acquired bacterial pneumonia: a global, double-blind, multicentre, randomised, active-controlled, non-inferiority trial (SOLITAIRE-ORAL). Lancet Infect Dis. 2016;16:421-30. https://doi.org/10.1016/S14733099(16)00017-7.

55. File TM, Rewerska B, Vucinić-Mihailović V, Gonong JRV, Das AF, Keedy K, et al. SOLITAIRE-IV: a randomized, double-blind, multicenter study comparing the efficacy and safety of intravenous-to-oral solithromycin to intravenous-to-oral moxifloxacin for treatment of community-acquired bacterial pneumonia. Clin Infect Dis. 2016;63:1007-16. https://doi.org/10.1093/cid/ciw490.

56. Chen MY, McNulty A, Avery A, Whiley D, Tabrizi SN, Hardy $\mathrm{D}$, et al. Solithromycin versus ceftriaxone plus azithromycin for the treatment of uncomplicated genital gonorrhoea (SOLITAIRE$\mathrm{U})$ : a randomised phase 3 non-inferiority trial. Lancet Infect Dis. 2019;19:833-42. https://doi.org/10.1016/S1473-3099(19) 30116-1.

57. Crandon JL, Nicolau DP. In vivo activities of simulated human doses of cefepime and cefepime-AAI101 against multidrug-resistant gram-negative enterobacteriaceae. Antimicrob Agents Chemother. 2015;59:2688-94. https://doi.org/10.1128/AAC.00033-15.

58. Crandon JL, Nicolau DP. In vitro activity of cefepime/AAI101 and comparators against cefepime non-susceptible enterobacteriaceae. Pathogens. 2015;4:620-5. https://doi.org/10.3390/patho gens 4030620 .

59. Allecra. Safety and efficacy study of cefepime-AAI101 in the treatment of complicated urinary tract infections-full text view-ClinicalTrials.gov 2018. https://clinicaltrials.gov/ct2/show/ NCT03687255. Accessed 15 Dec 2020.

60. Vickers RJ, Tillotson GS, Nathan R, Hazan S, Pullman J, Lucasti $\mathrm{C}$, et al. Efficacy and safety of ridinilazole compared with vancomycin for the treatment of Clostridium difficile infection: a phase 2 , randomised, double-blind, active-controlled, non-inferiority study. Lancet Infect Dis. 2017;17:735-44. https://doi.org/10.1016/ S1473-3099(17)30235-9.

61. Steinebrunner N, Stremmel W, Weiss KH. Ridinilazole-a novel antibiotic for treatment of Clostridium difficile infection. J Thorac Dis. 2018;10:118-20. https://doi.org/10.21037/jtd.2017.12.117.
62. Summit Therapeutics. A study of ridinilazole (SMT19969) compared with fidaxomicin for the treatment of Clostridium difficile infection (CDI) - full text view-ClinicalTrials.gov 2016. https:// clinicaltrials.gov/ct2/show/NCT02784002. Accessed 15 Dec 2020.

63. Summit Therapeutics. Comparison of ridinilazole versus vancomycin treatment for Clostridium difficile infection-full text view-ClinicalTrials.gov 2018. https://clinicaltrials.gov/ct2/show/ NCT03595553. Accessed 15 Dec 2020.

64. Taylor SN, Morris DH, Avery AK, Workowski KA, Batteiger BE, Tiffany CA, et al. Gepotidacin for the treatment of uncomplicated urogenital gonorrhea: a phase 2, randomized, doseranging, singleoral dose evaluation. Clin Infect Dis. 2018;67:504-12. https://doi. org/10.1093/cid/ciy145.

65. O'riordan W, Tiffany C, Scangarella-Oman N, Perry C, Hossain M, Ashton T, et al. Efficacy, safety, and tolerability of gepotidacin (GSK2140944) in the treatment of patients with suspected or confirmed gram-positive acute bacterial skin and skin structure infections. Antimicrob Agents Chemother. 2017. https://doi.org/ 10.1128/AAC.02095-16.

66. GlaxoSmithKline. A dose-ranging study evaluating the efficacy, safety, and tolerability of GSK2140944 in the treatment of uncomplicated urogenital gonorrhea caused by Neisseria gonorrhoeaefull text view-ClinicalTrials.gov 2014. https://clinicaltrials.gov/ ct2/show/NCT02294682. Accessed 15 Dec 2020.

67. GlaxoSmithKline. Dose-ranging study of GSK2140944 in the treatment of subjects with suspected or confirmed gram-positive acute bacterial skin and skin structure infections-full text view-ClinicalTrials.gov 2014. https://clinicaltrials.gov/ct2/show/ NCT02045797. Accessed 15 Dec 2020.

68. GlaxoSmithKline. A study to evaluate efficacy and safety of gepotidacin in the treatment of uncomplicated urinary tract infection (UTI) - full text view-ClinicalTrials.gov 2019. https://clini caltrials.gov/ct2/show/NCT04020341. Accessed 15 Dec 2020.

69. Barnes MD, Kumar V, Bethel CR, Moussa SH, O'donnell J, Rutter JD, et al. Targeting multidrug-resistant Acinetobacter spp.: sulbactam and the diazabicyclooctenone $\beta$-lactamase inhibitor etx 2514 as a novel therapeutic agent. MBio. 2019;10:1-15. https://doi.org/ 10.1128/MBIO.00159-19.

70. Entasis Therapeutics. Study to evaluate the efficacy and safety of intravenous sulbactam-ETX2514 in the treatment of patients with infections caused by Acinetobacter baumannii-calcoaceticus complex-full text view-ClinicalTrials.gov 2019. https://clini caltrials.gov/ct2/show/NCT03894046. Accessed 15 Dec 2020.

71. Kern G, Palmer T, Ehmann DE, Shapiro AB, Andrews B, Basarab GS, et al. Inhibition of Neisseria gonorrhoeae type II topoisomerases by the novel spiropyrimidinetrione AZD0914. J Biol Chem. 2015;290:20984-94. https://doi.org/10.1074/jbc.M115.663534.

72. National Institute of Allergy and Infectious Diseases (NIAID). Randomized, open-label phase 2 study of oral AZD0914 in the treatment of gonorrhea-full text view-ClinicalTrials.gov 2014. https://clinicaltrials.gov/ct2/show/NCT02257918. Accessed 15 Dec 2020.

73. VenatoRx Pharmaceuticals I. Safety and efficacy study of cefepime/VNRX-5133 in patients with complicated urinary tract infections-full text view-ClinicalTrials.gov 2019. https://clini caltrials.gov/ct2/show/NCT03840148. Accessed 15 Dec 2020.

74. Tacconelli E, Magrini N. Global priority list of antibiotic-resistant bacteria to guide research, discovery, and development of new antibiotics. Organ Mund La Salud. 2017. pp. 1-7. http://www.cdc. gov/drugresistance/threat-report-2013/. Accessed 20 Feb 2021.

75. Tacconelli E, Carrara E, Savoldi A, Harbarth S, Mendelson M, Monnet DL, et al. Discovery, research, and development of new antibiotics: the WHO priority list of antibiotic-resistant bacteria and tuberculosis. Lancet Infect Dis. 2018;18:318-27. https://doi. org/10.1016/S1473-3099(17)30753-3. 
76. M P, L S. Clinical research designs to study treatment effects for multidrug-resistant bacteria. Clin Microbiol Infect 2019;25:92931. https://doi.org/10.1016/J.CMI.2019.05.004.

77. JA M, JP D, GH T, LE C, I F, A S, et al. Plazomicin for infections caused by carbapenem-resistant enterobacteriaceae. N Engl J Med 2019;380:791-3. https://doi.org/10.1056/NEJMC1807634.

78. M P, V S, E M, G K, E R, L L. Systematic review and meta-analysis of the efficacy of appropriate empiric antibiotic therapy for sepsis. Antimicrob Agents Chemother 2010;54:4851-63. https:// doi.org/10.1128/AAC.00627-10.
79. Cama J, Leszczynski R, Tang PK, Khalid A, Lok V, Dowson CG, et al. To push or to pull? In a post-COVID world, supporting and incentivizing antimicrobial drug development must become a governmental priority. ACS Infect Dis. 2021. https://doi.org/10. 1021/acsinfecdis.0c00681.

80. Yahav D, Shepshelovich D, Tau N. Cost analysis of new antibiotics to treat multidrug-resistant bacterial infections: mind the gap. Infect Dis Ther. 2021. https://doi.org/10.1007/ s40121-021-00412-y.

\section{Authors and Affiliations}

\section{Jaffar A. Al-Tawfiq ${ }^{1,2,3,18}$ (D) Hisham Momattin ${ }^{4} \cdot$ Anfal Y. Al-Ali $^{5} \cdot$ Khalid Eljaaly $^{6,7} \cdot$ Raghavendra Tirupathi $^{8,9,10}$. Mohamed Bilal Haradwala ${ }^{11}$. Swetha Areti ${ }^{12}$. Saad Alhumaid ${ }^{13}$. Ali A. Rabaan ${ }^{14,19}$. Abbas Al Mutair ${ }^{15,16,20}$. Patricia Schlagenhauf ${ }^{17}$}

1 Specialty Internal Medicine and Quality Patient Safety Department, Johns Hopkins Aramco Healthcare, Dhahran, Saudi Arabia

2 Infectious Diseases Division, Department of Medicine, Indiana University School of Medicine, Indianapolis, IN, USA

3 Infectious Diseases Division, Department of Medicine, Johns Hopkins University School of Medicine, Baltimore, MD, USA

4 Department of Pharmacy Services, Mouwasat Hospitals, Dammam, Saudi Arabia

5 Department of Pharmacy Services, Dhahran Eye Specialist Hospital, Dhahran, Saudi Arabia

6 Faculty of Pharmacy, King Abdulaziz University, Jeddah, Saudi Arabia

7 College of Pharmacy, University of Arizona, Tucson, AZ, USA

8 Department of Medicine, Penn State College of Medicine, Hershey, PA, USA

9 Keystone Infectious Diseases/HIV, Keystone Health, Chambersburg, PA, USA

10 Department of Medicine, Wellspan Chambersburg and Waynesboro (Pa.) Hospitals, Chambersburg, PA, USA
11 Department of Neurology, University of Missouri Hospital, Columbia, Missouri, USA

12 Department of Hospital Medicine, Wellspan Chambersburg and Waynesboro (Pa.) Hospitals, Chambersburg, PA, USA

13 Administration of Pharmaceutical Care, Alahsa Health Cluster, Ministry of Health, Alahsa, Saudi Arabia

14 Molecular Diagnostic Laboratory, Johns Hopkins Aramco Healthcare, Dhahran, Saudi Arabia

15 Research Center, Almoosa Specialist Hospital, Al-Ahsa, Saudi Arabia

16 School of Nursing, University of Wollongong, Wollongong, Australia

17 WHO Collaborating Centre for Travellers' Health, Institute for Epidemiology, Biostatistics and Prevention, University of Zürich Centre for Travel Medicine, Zurich, Switzerland

18 Dhahran Health Center, Johns Hopkins Aramco Healthcare, P.O. Box 76; Room A-428-2, Building 61, Dhahran 31311, Saudi Arabia

19 Department of Public Health and Nutrition, The University of Haripur, Haripur 22610, Pakistan

20 College of Nursing, Princess Norah Bint Abdulrahman University, Riyadh 12214, Saudi Arabia 